\title{
El glossari contingut en el manuscrit París, Bibl. Nat. lat. 2306 *
}

\author{
Jesús Alturo i Perucho
}

El manuscrit lat. 2306 de la Biblioteca Nacional de París no sembla haver atret gaire l'atenció dels estudiosos ', i, tanmateix, té per als catalans un interès remarcable, car possiblement cal buscar el seu origen en algun escriptori de la nostra terra ${ }^{2}$. I és que aquest còdex ingressà a la biblioteca parisina a través de la rica col-lecció J. B. Colbert, que, com és sabut ${ }^{3}$, recolli molts manuscrits d'arreu de França i també d'origen estranger, $i$, entre aquests no faltaren els catalans, que després passaren a la biblioteca del rei, més endavant nacional ${ }^{4}$. Però si aquest simple detall no pot ésser argüit, naturalment, com a concloent amb vista a

\footnotetext{
* Aquest treball ha estat possible gracies a un ajut de la Comissió Interdepartamental de Recerca i Innovació Tecnologica, CIRIT.

' De tet, que jo sàpiga, no ha estat mai objecte d'un estudi monogràfic. Amb tot, se'n serveixen indirectament H. M. RoCHAIS, "Contribution à l'histoire des florilèges ascétiques du haut moyen àge", Revue Bénédictine, 63 (1953), pág. 246, i C. M. BatLle, "Die "Adhortationes sanctorum Patrum" im Lateinischen Mittelalter", dins "Beitrage zur Geschichte des Alfen Mönchtums und des Benedektinesordens". Heft, 31, Münster 1972, pág. 147, i l'esmenta R. WASSELYNCK, "Les compilations des "Moralia in lob" du vil" au XI" siecle, Recherches de Théologie Ancienne et Médievale, 29 (1962), pág. 10. Naturalment, també és considerat en el Catalogue général de manuscrits latins, II, Paris 1940, pág. 398.

2 Aquesta és també l'opinió de G. Colon-A. J. Soberanas, Panorama de la lexicografia catalana, Barcelona 1985, pág. 12, n. 4, en esmentar el glossari copiat en un full en blanc del manuscrit del qual tractaré.

${ }^{3}$ Sobre la magnifica biblioteca de J. B. Colbert, una de les millors col leccions privades de l'Europa del segle XVII, vegeu L. DeLISLE, Le Cabinet de Manuscrits de la Bibliotheque Impériale. Étude sur la formation de ce dépôt, Paris 1868, I, págs. 439-486; l'exposició Colbert, 1619-1683, Paris 1983, i Ch. DE LA RonCIERE-P.-M. Bondols, Catalogue des manuscrits de la collection des Mélanges Colbert, Paris 1920.

${ }^{4}$ Per a la seva història, vegeu ara S. BALAYE, La Bibliothèque Nationale des origines à 1800, Geneve 1988.
} 
determinar aital origen, sí hom pot adduir amb més força de prova les particularitats paleogràfiques de les tres mans ${ }^{5}$ que copiaren les Sententiae de Taió de Saragossa, obra que transmet el còdex i de la qual, d'altra banda, ja coneixíem una primerenca i significativa difusió per Catalunya ${ }^{6}$. Aquestes tres mans, en efecte, se serveixen d'una escriptura minúscula carolina, no exempta de notables reminiscències visigòtiques $^{7}$, ço que és propi de l'escriptura librària de final del segle ix emprada al nostre país ${ }^{8}$. Crec, doncs, que un hom pot atribuir amb una certa seguretat a un centre català i a final de l'esmentada centúria, i, més en concret, a una data propera a l'any 900 o un xic anterior ${ }^{9}$, la còpia del manuscrit que ens ocupa, la descripció codicològica del qual considero útil de consignar.

La pàgina, en pergami generalmente gruixut, amida $330 \times 230 \mathrm{~mm}$. La caixa d'escriptura $250 \times 170 \mathrm{~mm}$, és a dir que l'altura de la caixa correspon gairebé a l'amplària del foli i l'altura d'aquest és quasi el doble de l'emplària d'aquella. Escriptura a dos corondells, amb una estructura $2 v 2 v 2 v 2 v i 29$ línees per columna alternant amb una estructura $1 \mathrm{v} 1 \mathrm{v}$ $1 v$ 1v i 32 línees per columna ${ }^{10}$. La separació entre linees de guiatge és de $10 \mathrm{~mm}$ sense que la primera línea d'escriptura quedi mai enmarcada per dalt i l'altura mitja de les lletres fa $2,5 \mathrm{~mm}$. L'intercolumni és de $15 \mathrm{~mm}$. El marge superior té $30 \mathrm{~mm}$, l'inferior i el lateral exterior, $50 \mathrm{~mm}$, i l'interior, $15 \mathrm{~mm}$. Diverses marques de punxat de forma allargada són visibles als marges de la pàgina. El lineament a punta seca és assenyalat

\footnotetext{
5 Vegeu la nota 10

${ }^{6}$ En el testament dictat pel bisbe Sisebut d'Urgell el 28 de març de 839, aquest prelat llegà diversos llibres, entre ells, unes Sententiae de Taió deixades a Sant Climent de Codinet, cosa que sı no prova que aquest còdex fos copiat a Catalunya, si demostra que circulà pel nostre país, indici igualment evident de l'interes despertat per l'obra. Sobre la biblioteca de Sisebut $i$ altres notícies de llibres contingudes en diplomes d'Urgell, vegeu P. PuJOL I Tubau, "De la cultura catalana medieval. Una biblioteca dels temps romànics", Estudis Universitaris Catalans, 7 (1913), págs. 1-8, recollit també en Obra completa, Valls d'Andorra 1984, págs. 47-54; “La cultura pirinenca en l'Alta Edat Mitjana", Pirineos, 4 (1948), páginas 385-407, i en Obra completa, págs. 623-640, i «El manuscrit de la Vulgata de la Catedral d'Urgell", Butlletí de la Biblioteca de Catalunya, 6 (1920-1922), págs. 98-144, i en Obra completa. págs. 193-247.

$?$ Vegeu infra.

${ }^{8}$ Vegeu A. M. Mundo-J. ALturo, "La escritura de transición de la visigótica a la carolina en la Cataluña del siglo $X$ " dins Actas del VIII Coloquio del Comité Internacional de Paleografia Latina. Madrid-Toledo 1987, Madrid 1990, págs. 131-138. anterior.

Per als criteris de datació paleográfica, remeto a la ponència esmentada en la nota

${ }^{10}$ Els quaderns amb estructura $2 v$ són els $1,2.3,4,5,15,16,17,18$ i 19 , la resta presenten una estructura $1 \mathrm{v}$. A cada tipus de quadern correspon una mà de copista distinta, a les quals cal afegir una tercera mà que sols transcriu paràgrafs aillats.
} 
gairebé sempre damunt el costat pèl ${ }^{11}$, costat que inicia sense excepció els quaderns ${ }^{12}$; aquests estan numerats en el centre del marge inferior de la darrera pàgina ${ }^{13}$, trenta quaderns tenen una estructura de quaterns $i$ el darrer està format només per tres folis. Però el còdex sols té un total de 232 folis perquè n'ha perdut alguns ${ }^{14}$. El lineament és marcat en general bifoli per bifoli, però alguna vegada també de dos bifolis en $\operatorname{dos}^{15}$.

Pel que fa al tipus d'escriptura de les tres mans principals del còdex ja he dit que és la minúscula carolina amb notabilíssimes reminiscències de la visigòtica, com és ara l'ús de la $g$ uncial ${ }^{16}$, de la a oberta ${ }^{17}$, de la I alta ${ }^{18}$, de la $e$ dreta ${ }^{19}$, del signe d'abreviatura de nasal constituilt per una ratlla sobremontada per un punt ${ }^{20}$, de l'abreviatura d'algun possessiu $^{21}$ i de la mateixa ortografia amb l'ús incorrecte de la $h^{22}$, la confusió $u / b^{23}, i / e^{24}$ o l'ús d'un sistema abreviatiu indecís i poc

11 Excepte en els quaderns 10 i 11.

${ }_{12}$ Els quaderns que actualment comencen amb el costat carn -1, 11, 14, 16 i 19- han perdut el primer foli. Vegeu la nota 14.

${ }_{13}$ Alguna vegada aquesta numeració, en números romans precedits d'una $q$, no es veu - en els quaderns $1,2,3$ i 15-, perquè una mà correctora que actua cap al 1200 ha esborrat alguns comentaris marginals d'una altra ma correctora i anotadora, coetània de la còpia del còdex, i amb aquestes anotacions ha desaparegut també la numeració dels quaderns.

${ }_{14}$ Falta un foli entre els actuals $18-19,97-98,100-101,113-114,136-137,185-186$ i 191192.

${ }^{15}$ En els quaderns 2 i 9.

${ }^{16}$ Vegeu gloria (fol. 33r) o neglegentiae corregit negligentiae (fol. 43r).

17 Usa les a obertes sobretot la mà de menor activitat en la copia del text; vegeu mansionibus, quamuis, aeternis, solari (fol. 3v); creatori (fol. 13v); purgato, illa, caelibus (fol. 42r); mane, Adam, tertia (fol. 111v). Pero de vegades també en fan ús esporàdicament les dues mans principals; vegeu actibus (fol. 14v); laudes (fol. 115r); intra, caritatis, sitam, iura, lapide, nostra (fol. 115v); hobaedit corregit obaedit (fol. 143r); temperatur, poterant (fol. 152r); consumta corregit consumpta, securitas (fol. 154r); liberatus, contra, factum, propheta (fol. 165r).

${ }_{18}$ Sovint la i és semialta, però alguna vegada completament alta, com en ld est corregit id est (fol. 159r).

${ }_{19}$ Vegeu saeculo (fol. 34r), unumquemque (fol. 165v), est (fol. 206r).

${ }^{20}$ Vegeu superbientium (fol. 16r); repellendum (fol. 158v); summopere (fol. 185r)

21 Vegeu noster (fol. 129v, 135r, 160v).

${ }^{22}$ Sovint aquest ús incorrecte de la $h$ és corregit; vegeu hid >id (fol. 1v, 213r), abere>habere (fol. 3r), hortum>ortum (fol. 10v), ostes>hostes (fol. 51r), abebit>habebit (fol. 77r), inuli > hinnuli (fol. 77v), umore >humore (fol. 98r), hodio >odio (fol. 120v), hobaedit>obaedit (fol. 143r), hosteum>ostium (fol. 15tv), peribit>perhibet (fol. 182r). Algunes vegades, però, la forma incorrecta no ha estat esmenada; vegeu hictibus (fol. 45r), orribilis (fol. 120v), toth (fol. 127v), habundantes (fol. 147r), hutilitas (fol. 151v), habundantia, Habrahae (fol. 230v).

${ }_{23}$ Aquests errors també són esmenats sovint; vegeu tauernaculo >tabernaculo (fol. 2r), preuemus $>$ prebemus (fol. $80 \mathrm{r}$ ), dilubium $>$ diluuium (fol. $84 \mathrm{v}$ ), inuecillitatem $>$ inbecillitatem (fol. 85 r).

${ }^{24}$ Aquesta confusió és també corregida; vegeu pinnae $>$ pennae (fol. 77r), cortix $>$ cortex 
constant ${ }^{25}$, característiques totes aquestes $\mathrm{i}$ altres ${ }^{26}$ que ens permeten de situar la còpia del manuscrit un poc abans de l'any 900 i en terra catalana, on ja trobem bons exemples de la substitució de l'antiga escriptura visigòtica per la nova continental, en la modalitat librària, cap a l'any 880 sobretot per a la còpia de diplomes d'una certa solemnitat i de còdexs $^{27}$.

Però, ultra les tres mans que en diferent mesura dugueren a terme la transcripció de la coneguda obra tajoniana, hi intervingueren dues altres de semblants característiques paleogràfiques, i, per tant, coetànies, a les quals hom deu distintes correccions en el text i diverses anotacions marginals ${ }^{28}$. D'aquestes dues mans correctores $i$ anotadores -a les quals caldria sumar una tercera, de tinta més fosca i de cap a l'any $1200^{29}$-, n'hi ha una de més activa i pulcra ${ }^{30}$, que copià en un foli en blanc ${ }^{31}$ un petit glossari constituït per 48 lemmata que comencen sols amb la lletra $D$ i mantenen un ordre força alfabètic ${ }^{32}$, de les dues prime-

(fol. 77v), dispexit $>$ despexit (fol. 78v), discriptam>descriptam (fol. 92r), hosteum>ostium (fol. 151v).

${ }_{25}$ Vegeu, per exemple, diverses maneres d'escriure autem: autem (fol. $7 \mathrm{~V}$ ), aum (fol. $9 \mathrm{v}, 10 \mathrm{r}, 14 \mathrm{v}$ ), aut (fol $19 \mathrm{r}, 21 \mathrm{v}$ ), aute (fol. 80v), au (fol. 139r); o namque: naq (fol. 89v), namq (fol. 92v) o apostolus: apls (fol. 39v), apostolus (fol. 45v), aps/s (fol. 124v), aps/us (fol. 129v).

${ }^{26} \mathrm{Com}$, per exemple, l'abreviatura per a Israhel:Srhl (fol. 2r. 204r), Srl (fol. 6r); l'ús de $q$ en lloc de c: quur (fol. 8r), quoram (fol. 155r), o a l'inrevés: anticus (fol. 193r), derelincunt (fol. 199r), quohercuit corregit cohercuit (fol. 83r); la $u$ sobreposada damunt $q$ : aequus corregit equus (fol. 189r), que (fol. 208v); determinats nexes, on, ultra els usuals ct i st, són freqüents els formats per $r$ seguida de consonant: $t$-infertur (fol. $1 \mathrm{v}$ )-, $m$-aeternam (fol. 118v)-, rm -infirmitatibus (fol. 119v) - $r c$-exorcismi (fol. 126r) - , rr -narrant (fol. 126r)-, rs --uersus (fol. 127v) - , rg -purgat (fol. 188r)-; o per $r$ seguida de vocal ro -uestro (fol. 192v) - re -timore (fol. 193r) -; el nexe es -destruendum (fol. 200v)-, i nt -deficient (fol. 223v)-. Aquí, per últim, també podem incloure la paraula ecclesia escrita amb una sola $c$ (fol. 126r, 127v).

${ }^{27}$ Vegeu la nota 8.

${ }^{28}$ En aquestes notes marginals hom troba citacions textuals extretes de sant Agusti, de sant Ambros, de Baquiari, de sant Isidor i de les Vitae Patrum, indici indirecte de la circulació de les obres d'aquests autors per Catalunya.

29 Aquesta mà acostuma a esborrar anotaciuons anteriors - vegeu la nota $13-i$, entre altres esmenes, interposa una $p$ entre $m$ i $n$.

${ }_{30}$ Aquesta altra mà no sols anota i corregeix el text sinó també la morfologia i l'ortografia. Aixi té tendencia a allargar la base de les $i, r, s$ i $x$, i també posa accents, aguts (septuagésimi, fol. 1v) i circumflexs (formidat, fol. 1v). Pel que fa a les seves caracteristiques paleogràfiques, cal ressaltar l'ús del nexe /i (mulieribus, fol. $2 r$ ) i de $t i$, independentment del seu valor sibilant (oratio, fol. $3 r$ ) o dur (electi, fol. $3 \mathrm{v}$ ); també se serveix de la $o$ amb cresta, de tradició cursiva (pongere, fol. $2 \mathrm{v}$ ), de les $e$ dretes (poenitentiam, fol. $2 \mathrm{r}$ ) i de la ratlla amb un punt al damunt per indicar abreviatura de nasal (postmodum, fol. 38r; speciem, fol. 103v). a més dels accents aguts i circumflexs ja comentats.

${ }^{31}$ El foli $106 \mathrm{r}$.

32 Aquest ordre es trenca en les glosses $21,23,24,26,28,31,32,36,38,40,44$ i 46 
res lletres com a minim. A aquestes glosses cal afegir-hi sis més, que, degudes a la mateixa mà, hom troba escampades pels marges laterals del còdex, el qual, com he assenyalat, acull sovint comentaris i notes diverses.

Les fonts del nostre glossari el fan molt pròxim al del tipus anomenat Abavus ${ }^{33}$, puix que setze de les quaranta-vuit glosses corresponen amb total exactitud a aquest glossari $(1,2,4,5,7,8,13,14,19,23,24,27$, $29,30,31$ i 45); altres vuit són també iguals, bé que augmentades amb algun nou afegit explicatiu $(11,16,17,20,22,25,40$ i 43), i dues són solament semblants ( $9 \mathrm{i}$ 15). Les glosses no' contingudes en Abavus-vinti-dues $(3,6,10,12,18,21,26,28,32,33,34,35,36,37,38,39,41$, $42,44,46,47 ; 48)$, en alguns casos són idèntiques o gairebé a altres glossaris: en vuit ocasions a l' $A a^{34}(18,21,26,37,39,41,42$ i 44) i en tres pròximes a aquest glossari $(9,15 \mathrm{i} 17)$; en altres vuit casos són iguals o molt semblants a l'Ansileubus ${ }^{35}(12,18,21,33,34,37,39$ i 44) i una vegada pròxima a aquest glossari (9); en sis casos són iguals a $A_{b b a}{ }^{36}(3,26,39,41,42 i 44)$ i en tres pròximes a aquest glossari (15, 16 i 17); en quatre casos iguals o quasi a $\operatorname{Abstrusa}^{37}(12,18,37$ i 41); dues vegades iguals o gairebé a $A_{b o l i t a}{ }^{38}$ (38 i 40) i una pròxima a aquest glossari (11); i, finalment, en dues altres ocasions són quasi iguals al glossari Arma ${ }^{39}$ (37 i 39). Curiosament una glossa, la 46 , hom troba en Abó de Saint-Germain, autor del poema Bella Parisiacae urbis, compost amb mots tan rars que es veu obligat a explicar-los ${ }^{40}$. Només resten sense font literal o aproximada coneguda quatre glosses $(6,32,36$ i 47) ${ }^{11}$.

33 Hom el trobarà editat per J. F. MOUNTFORD dins Glossaria Latina, II, París 1926 , páginas 23-121.

34 Editat per C. Theander dins Glossaria Latina, V. Paris 1931, págs. 145-388.

35 Edital per W.-M. Lindsay, J.-F. Mountford, J. Whatmough, F. Rees, R. Weir, M. LAISTNER dins Glossaria Latina, I, Paris 1926.

${ }^{36}$ Editat per M. Inguanez, C.-J. Fordyce dins Glossaria Latina, V, París 1931, págs. 7143.

37 Editat per W.-M. Lindsay, H. J. Thomson dins Glossaria Latina, III, París 1926.

38 Editat per W.-M. LindSay dins Glossaria Latina, Ill, Paris 1926, págs. 91-183

39 Editat per R.-G. Austin, W.-M. Lindsay dins Glossaria Latina, Paris 1926, págs. 1-22.

40 Vegen els versos 16-17: Amphytappa laon extat badanola, necnon Effipiam diamant, stragulam pariterque propomam, dins Monumenta Germaniae Historica, Poetae Latini aeni Carolini. IV, págs. 116 y sgtes., on hom explica que diamant significa ualde amant.

41 Altres quatre glosses tenen una font coincident amb glossaris editats per G. LOEWEG. Goetz, Corpus glossariorum Latinorum, 7 vols., Leipzig 1888-1901; Excerpta ex glossis Aynardi (10) -vegeu LOEWE-GoEtz, V, 618, 23-; Glossarium Amplonianum secundum -Loewe-Goetz, V, 285, 1-; I specimen codicis Cantabr. Collegii Corp. Chr. -LoewEGoetz, V, 406, 74-; Glossae Scaligeri-LoeWE-Goetz, V, 596, 52- (28); Glossae. Graeco- 
Per tant, si afegim aquest glossari als altres que ja coneixem del mateix origen i semblant data ${ }^{42}$, disposarem per a la Catalunya del segle IX d'un grup gens negligible de quatre glossaris, més un altre text de caràcter gramatical: un Donat actualment custodiat a l'Arxiu Capitular de Toledo ${ }^{43}$. Tot fa pensar, doncs, que a la Catalunya de la novena centúria, $\mathrm{o}$, si hom vol a la protoCatalunya millor que no pas pre-Catalunya d'aquesta època ${ }^{44}$, l'estudi de la gramàtica no fou descurat, almenys a final de segle, tal com, d'altra banda, queda reflectit en una lleugera milloria del modest coneixement de llatí que evidencia l'anàlisi dels textos $i$ documents conservats, respecte al llati més deturpat de mitjan segle, que representa un descens en relació al grau de coneixements gramaticals de començos de la centúria ${ }^{45}$

Dono tot seguit la transcripció del glossari i en apèndix les glosses marginals.

1 Dedicationes: encenia.

Deditio: traditio spontanea.

Dedunt: tradunt.

Defungitur: moritur.

latinae (35) -Loewe-Goetz, II, 269, 2-; Glossae Ab absens (48) - LoeWE-Goetz, IV, 409, $35-$.

D'altra banda, de les sis glosses marginals sols tres tenen una font exacta (Appendix 2) o aproximada (Appen. 4 i 5), que en dos casos coincideix també amb Abavus.

${ }_{42}$ La llista completa hom pot veure en J. ALTURO, "Els manuscrits i documents llatins d'origen català de! segle Ix", dins les Actes del Symposium internacional sobre els origens de Catalunya, Barceiona 1989 (en prensa). L'estudi i edició de tots aquests glossaris penso incorporar-los dins el Corpus glossariorum Latinorum Cataloniae (saec. IX-XII) que preparo.

${ }_{43}$ Es tracta del manuscrit 99-30. Vegeu L. HoLtz, Donat et la tradition de l'enseignement grammatical. Étude et édition critique, Paris 1981, págs. 384-386.

${ }_{44}$ Si per mor de precisió s'ha de filar prim en la terminologia emprada per referir-nos a la coneguda, llarga i purista perifrasi "el territori que amb el temps esdevindrà Catalunya» i si per a aquest proposit no és suficient un simple article determinat, creuria més convenient de parlar de protoCatalunya abans que de pre-Catalunya - denominació emprada per R. d'Abadal i seguida després per altres historiadors-, car considero que aquest terme no inclou necessàriament - $i$, en certa mesura, exclou - el concepte de la Catalunya incipient, que si no tenia encara el nom actual, almenys la seva realitat es feia present en els seus habitants, que al segle $1 \times$ se servien ja d'una parla que, com diu el professor J. BASTARDAS, "Quan es produí el pas del liatí al català?", Revista de Catalunya, 30 (1989), págs. 33-47, no hem de tenir por a qualificar de català.

${ }^{45} \mathrm{Vegeu}$ al respecte J. Alruro, El coneixement del llati en la Catalunya del segle IX (en curs de publicació). 
5 Deierent: iurent.

De interitu: de morte.

Delibatio: $<\mathrm{p}>$ refloratio, immolatio.

Delibor: immolor.

Delibitum: diligenter unctum.

10 Delticus: licteratus (litt-).

Delubrum: templum, ara, fanum, idola, puluinar uel quicquid $<$ in idolo colitur $>$.

Demetiar: diuidam.

Demptio: separatio.

Depositio: obductio uel collocatio.

15 Desapit: delirat.

Desecto: inciso, decollato.

Desidia: neglegentia, ignauia.

Despiciens: desuper aspiciens.

Despondit (-det): ualde promittit.

20 Destitutum: relictum, desolatum, destructum.

De stipite: de ligno.

Desuper: super, supernae uel ex $<s>$ uperiore parte.

Detectus: publicatus.

Desideratus: necessarius.

25 Detestabilis: pessimus, deterrimus, abominabilis, ex<s>ecrabilis, maledictus.

Deterrimum: de malo peiore.

Detractio: blasphemia uel reprehensio.

Dextralia: brachialia.

Detrusus: inmissus, inpulsus, inclusus.

30 Deuium: absque uia.

Deuirginat: corrumpit.

Delictio: oratio, oboedientia obsequio.

Deuterogamie: secunde nuptiae.

Deuteronomium: iteratio, doctrina, id est secunda lex.

35 Deuterosin: nouitatem.

Deus recens: deus nouus.

Dextra: prospera, sinistra, aduersa.

Dextimum: dextrum.

Dextrum: prosperum.

40 Diadema: uitta capitis regis uel corona.

Diabolus: criminator. 
Diaconus: minister.

Dialogus: disputatio uel liber disputationis, capituli dictio delectabilis uel duorum fabula.

Dialecticus: disputator uel genus philosophorum.

45 Dial<og>us: liber.

Diamat: ualde amat.

Diafonia: disponentia.

Dialabis: constitutum imperatorum.

\section{Appendix}

1 Deieiuno uel abstinentia (fol. 119r).

Histriones dicuntur saltatores, scenici (fol. 146v).

Egyptus interpretatur tribulatio (fol. 229r).

Theoria id est contemplatio Dei (fol. 230r).

5 Aequum hoc est rectum siue equalem (fol. $4 \mathrm{v}$ ).

Te igitur hoc est te certe (fol. $4 \mathrm{v}$ ).

1. Abavus DE 31// 2. Abavus DE 33, Abstr. DE 92, Ansil. DE 282// 3. Abba DE 20// 4. Aa D 104, Abavus DE 49// 5. Aa D 121, Abavus DE 58, cf. Abstr. DE 129, Arma D 213.

7. Cf. Aa D 163, Abavus DE 74// 8. Aa D 153, Abavus DE 75, cf. Abba DE 3, Arma D 206// 9. Cf. Aa D 537, cf. Abavus DE 76, cf. Abba DE 4, cf. Abstr. DE 52, cf. Ansil. DE 632// 10. LOEWE-GOETZ V 618, 23// 11. Cf. Aa D 161, cf. Abavus DE 89, cf. Abba DE 189, cf. Abol. DE 100, cf. Arma D 156, cf. Ansil. DE 688// 12. Abstr. DI 37, Ansil. DI 474// 13. Aa D 173, Abavus DE 95// 14. Abavus DE 111// 15. Cf. Aa D 297, cf. Abavus DE 138, cf. Abba DE 140// 16. Cf. Aa D 323, cf. Abavus DE 127, Abba DE 148// 17. Cf. Aa D 295, cf. Abavus DE 133, cf. Abba DE 90// 18. Cf. Aa D 300b, cf. Abstr. DE 100, cf. Ansil. DE 1107// 19. Abavus DE 142, Abstr. DE 108, Ansil. DE 1111// 20. Cf. Aa D 345, cf. DE 145// 21. Aa D 348, Ansil. DE 561// 22. Cf. Abavus DE 148// 23. Aa D 377, Abavus DE 150// 24. Abavus DE 131//25. Cf. Aa D 357, cf. Abavus DE 155, cf. Abba DE 60, cf. Abol. DE 155, cf. Ansil. DE 1182// 26. Cf. Aa D 361, Abba DE 35// 27. Abavus DE 162// 28. LOEWE-GoeTZ V 285, 1; 406, 74; 596, 52// 29. Cf. Aa D 285, Abavus DE 168, cf. Abba DE 88, cf. Abstr. DE 29, cf. Ansil. DE 1225// 30. Aa D 397, Abavus DE 178, cf. Abba DE 44, cf. Abstr. DE 112, cf. Ansil. DE 1277// 31. Abavus DE 176// 33. Cf. Ansil. DE 1333// 34. Cf. Ansil. DE 1336-1337// 35. LOEWE-GOETZ II 269, 2// 37. Aa D 429, cf. Abstr. DE 118, cf. Arma D 74, cf. Ansil. DE 1349// 38. Abol. DE 23// 39. Aa D 428, Abba DE 68, cf. Arma D 184, cf. Ansil. DE 1365// 40. Cf. Aa D 433, cf. Abavus DI 1, cf. Abol. DI 34, cf. Arma D 
154, cf. Ansil. DI 8// 41. Cf. Aa D 436, cf. Abba DI 119, Abstr. Dl 23b// 42. Aa D 437, Abba DI 8// 43. Cf. Aa D 439, cf. Abavus DI 2, cf. Abba DI 101, cf. Abstr. DI 37, cf. Ansil. DI 26// 44. Cf. Aa D 441, cf. Abba DI 102, cf. Abol. DI 54, Ansil. DI 19 i DI 18// 45. Aa D 438, Abavus DI 3// 48. Loewe-Goetz IV 409, 35.

Appendix: 2. Abavus HI 27// 4. Cf. Loewe-Goetz IV 291, 9// 5. Cf. Abavus AE 21. 